\title{
RADIO OUTFLOWS AND THE ORIGIN OF THE NARROW LINE REGION IN SEYFERT GALAXIES
}

\author{
A. CAPETTI ${ }^{1}$, D.J. AXON ${ }^{1,2}$, F.D. MACCHETTO ${ }^{1,2}$ \\ AND W.B. SPARKS ${ }^{1}$ \\ 1 Space Telescope Science Institute - 3700, San Martin Drive, \\ Baltimore, MD 21218, U.S.A. \\ 2 Astrophysics Division, Space Science Department of ESA - \\ ESTEC, NL-2200 AG Noordwijk, The Netherlands
}

Seven Seyfert galaxies, Mrk 3, Mrk 78, Mrk 348, Mrk 6, Mrk 573, NGC 3393, IRAS 04210+0400 and IRAS 11058-1131 (all Seyfert galaxies type 2 expect Mrk 6 which is a Seyfert 1.5) were observed with HST. For the first five objects images were taken with the FOC with filters centered on the emission lines $[\mathrm{O} \mathrm{II}]$ and $[\mathrm{O} \mathrm{III}]$. For the remaining three galaxies WFPC2 observations were obtained in the [O III] and $\mathrm{H} \alpha$ lines.

These images allowed us to explore the morphology of their NLRs which extend over less than $200 \mathrm{pc}$ in the case of Mrk 348 to more than $16 \mathrm{kpc}$ in IRAS 04210+0400. In Mrk 3, Mrk 6 and Mrk 348 the line-emission takes the form of a linear structure while in all the other galaxies series of spectacular arcs of line emission are present on both sides of the nucleus (see Fig. 1). In each case the radio and the line emission are very closely associated. In Mrk 3 and Mrk 348 UV $(\sim 2100 \AA)$ continuum emission has been detected and it appears to be cospatial with the radio jets.

The observed morphology of the NLR of these Seyfert galaxies can be explained if this results from the interaction of the radio ejecta with the surrounding gas. The line-emitting gas is compressed by the shocks created by the passage of the supersonic jets or by the sweeping-up of gas by the expanding radio lobes. The increase in the density due to the compression causes the line-emission to be highly enhanced in the region where this interaction occurs. The extended UV component may arise from free-free emission of the gas heated by the shocks formed by the radio jets. Ground based long-slit spectroscopy of these galaxies also shows the hallmark of this interaction in their nuclear regions, i.e. very broad and often splitted 

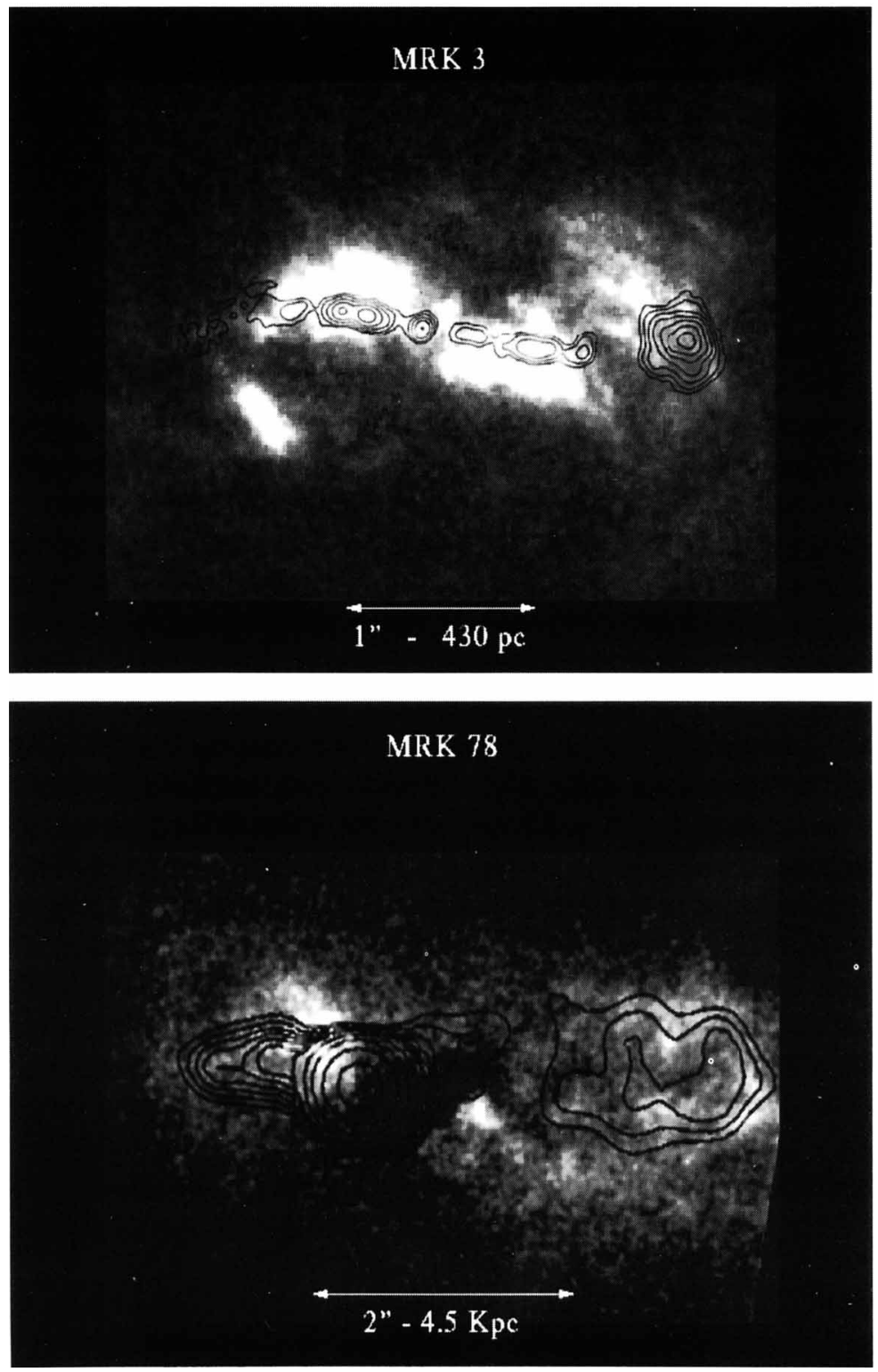

Figure 1. [O III] emission line image of two galaxies observed in this project (Mrk 3 and Mrk 78) with superposed the contour radio maps

lines. It therefore appears that the morphology, the dynamics and possibly the ionization structure of the NLR of these Seyfert galaxies is dominated by the effects of the radio outflows. 